\title{
5 Post-Diasporafilm oder Postdiaspora-Film? Ein Fazit
}

In Richard Linklaters Film Waking Life zeigt eine Film-im-Film-Szene - „The Holy Moment" - den Lyriker David Jewell mit dem Regisseur Caveh Zahedi in einer Interviewsituation. Beide sind durch die halbautomatische Technik der Vektorisierung rotoskopisch animiert und sprechen über André Bazins Kino-, Ästhetik- und Realismus-Begriff. Der Effekt der Film-im-Film-Szene wird durch die Rahmung der Leinwand und im weiteren Sinne durch die Rahmung des Kinosaales kreiert. Im Kinosaal wird das Interview vom Protagonisten (Wiley Wiggins) als Einzigem im Auditorium gesehen, das Bild ist in beiden Fällen stark stilisiert und erweckt den Eindruck, es würde auf- und abschwingen, als ob die Bewegungen der Figuren, ihre Mimiken und Gestiken, auch das Bild mitbewegen würden. Der dadurch kreierte filmische Raum entzieht sich festen $\mathrm{Zu}$ schreibungen, obwohl ihm eindeutig ein natürlicher Ursprung zugrunde liegt. Film sei, so führt Caveh Zahedi im Interview aus, hauptsächlich kein geschichtenerzählendes Medium:

If you tell a story or even like a joke: ,this guy walks into a bar and he sees a dwarf". That works really well because you're imagining this guy and this dwarf in the bar, and there's this kind of imaginative aspect to it. But in film you don't have that because you actually are filming a specific guy, in a specific bar, with a specific dwarf of a specific height, who looks a certain way, right? [...]. And so, it's about that guy, at that moment, in that space. ${ }^{64}$

Je mehr Film der narrativen Vorlage eines Drehbuches folgen würde, anstatt Ausdrücke und Handlungen der Darstellenden spontan aufzunehmen, umso weniger Platz sei für das, was er den ,heiligen Moment“ nennt, ein Moment der Öffnung, durch den Raum für Möglichkeiten und Gelegenheiten geschaffen 
wird. Die Szene endet mit der Verwandlung der zwei rotoskopischen Figuren in Wolken, gleichsam auf die Unbeständigkeit und Veränderlichkeit von Geschichten hinweisend. In diesem doppelten Wortsinn der sich ständig transformierenden Form der Wolke konstatierten Henry Jenkins, Sam Ford und Joshua Green in ihrer Theorie zur Verbreitung und Teilung von Geschichten im digitalen Zeitalter: „Perhaps nothing is more human than sharing stories, wherever by fire or by ,cloud““ (2-3) und verwiesen damit auf die cloud - die Wolke - als internetbasiertes Speicher- und Austauschmedium, gleichzeitig aber auch auf die Universalität von Geschichten und deren Erzählungen. Die Geschichten in den Filmen der iranischen Diaspora sind, wie in der vorliegenden Arbeit dargelegt werden konnte, geprägt von dieser Wandelbarkeit und erhöhten Medien- wie Raumsensibilität. Die Auseinandersetzung mit einem mehrdimensionalen Verständnis von Raum und Bewegung, welches sich nicht auf die tatsächliche physische Migration beschränkt, sondern Platz für (kosmo)feministische, dritte, hybride, interkulturelle, generische, autonome, verlebendigte, kollektive, gegenwärtige, transvergente, stille, laute, postmigrantische und rap-präsentative Räume schafft, verbindet all jene Gegensätze, Wiederstände und Kontraste (nicht zufällig ist die Hälfte der Filme in Schwarz-Weiß gedreht) dessen, was Diaspora als Lebenserfahrung ausmacht.

Doch was versteht mensch unter Diasporafilm überhaupt? Diese Frage schloss sich unmittelbar an die Ausgangsfrage an, wie Diaspora, und spezifischer iranische Diaspora, zu definieren ist. Was Filme der iranischen Diaspora ausmacht, ist nicht ohne die Diskussion, was die iranische Diaspora eigentlich ist, zu beantworten. Aufgrund der Breite, die der Begriff Diaspora an sich mittlerweile angenommen hat, wurde schnell deutlich, dass dies nicht so leicht zu klären ist. Denn einerseits scheinen sich hartnäckig Definitionen zu halten, die Diaspora 
nur in Relation zum Herkunftsland sehen. So seien Menschen, die in einer Diaspora leben, „entwurzelt“, „,deterritorialisiert“ und von einem stetigen Rückkehrwunsch in ein symbolisches oder reales „Heimatland“ getrieben. Andere Definitionen wiederum bezeichnen jegliche Formen globaler Migrationsbewegungen als Diasporaphänomen. Zwischen diesen beiden Polen habe ich meine Definition der iranischen Diaspora angesiedelt. Es handelt sich um eine Diaspora, die aus einer Migrationsbewegung entstanden ist, verschiedene Generationen, Ethnien, Religionen und soziale Schichten umfasst und dadurch in ihrer Heterogenität keine abschließende Definition zulässt. Die Arbeit konnte jedoch aufzeigen, dass der Begriff ,iranische Diaspora“, in Abgrenzung zu verwandten Begriffen wie Exil, eine rezente (Selbst-)Beschreibung darstellt, die das Symptom der Prozesshaftigkeit des Begriffes selbst reflektiert: Ausgehend von Herangehensweisen, die Raum und Kultur als nicht-monolithische Größen auffassen, wird dabei Ethnizität als Marker zur Positionierung und Verortung aufrechterhalten. Der Diasporafilm nimmt hierbei gesteigert Verflechtungen, Wechselwirkungen und Transformationsprozesse in den Blick. Denn Diasporafilme sind Filme, die nicht nur außerhalb eines Ursprungsortes produziert und rezipiert werden, sondern Filme, die in einem ständigen kulturellen Austausch stehen. Sie sind hybride Amalgame stilistischer, sprachlicher, generischer, narrativer und ästhetischer Vielfalt. Die Filmschaffenden schöpfen zwar aus einem autobiographischen Fundus an Erfahrungen, Diasporafilm verfolgt jedoch mehr als einen therapeutischen Selbstzweck. Die Filme überschreiten transnationale filmische und kulturelle Grenzen, sowohl in Produktion wie auch in Distribution und Rezeption. Diaspora und Film bedingen und regen sich gegenseitig an, wodurch Diaspora vornehmlich als Medienraum zu begreifen ist. Wesentlich ist hierbei das Moment der kulturellen Differenz, das ermöglicht, Diasporafilm als eigenständige Filmkultur abseits von Ethnozentrismus und Kulturessentialismus zu begreifen, 
der filmische Räume hervorbringt, die einen multiperspektiven Umgang mit Nationalität, Ethnie, Religion, Sprache und Generation haben.

Im filmhistorischen Kontext der langen Tradition des iranischen Filmschaffens betrachtet, ist Diaspora nicht nur außerhalb, sondern auch innerhalb Irans ein Gegenstand. Das Filmschaffen außerhalb Irans ist hierbei in mannigfaltiger Art und Weise mit vergangenen und aktuellen Ereignissen in Iran verbunden, rekonfiguriert seine kulturelle Verortung dabei jedoch immer wieder neu und stellt dementsprechend kein Auswärts, sondern ein Inmitten dar. Zur Verhandlung von Diaspora in Filmen, die innerhalb Irans produziert wurden, ließe sich eine weitere Forschungstätigkeit anschließen.

Diasporafilm, so der Befund in der vorliegenden Arbeit, ermöglicht ein anderes Nachdenken sowohl über Kultur als auch über Raum und Film. Wenn Diasporafilm also nicht nur ein Forschungsobjekt, sondern auch eine Forschungsperspektive darstellt, so ermöglicht es meine Arbeit, weitere Filme unter den herausgearbeiteten Gesichtspunkten zu untersuchen. Die vorliegende Arbeit begreift sich demnach als Beginn der Untersuchung jener filmischen Formen, die nicht eindeutig in das Muster „Diasporafilm“ fallen. Zugleich soll diese Arbeit auch ein innovativer Beitrag zu einem erweiterten Verständnis im Hinblick auf die Kopplung von Diaspora, Raum und Film sein. Diaspora als Medienraum, dem kulturelle Verortung und filmische Transformationsprozesse eingeschrieben sind, ist demnach als Kontaktzone zu begreifen, die Rückschlüsse auf gesamtgesellschaftliche Phänomene zulässt. Diese filmischen Transformationsprozesse, verstanden als kulturelle Praktiken, ermöglichen eine dreifache Betrachtungsweise: Die erste umfasst eine vertikale Betrachtungsweise, in der Diasporafilm durch seine historische Abfolge und Kontextualisierung, sowohl als Diskurs, aber auch als Motiv in den Filmen ausgezeichnet ist. Darin kommt ihm die Funktion der Anordnung von Räumen abseits nationaler und kultureller Grenzen zu. 
Diasporafilm überschreitet dabei nationale Grenzen und beeinflusst dadurch sowohl nationale wie transnationale populäre filmische Räume. Die zweite Betrachtungsweise ist horizontal zu begreifen: Der Diasporafilm transformiert auf mannigfaltige Art und Weise den filmischen Raum selbst und birgt so das Potenzial für stilistischen, ästhetischen und formalen Wandel, womit wiederum ein politischer Appell verknüpft ist. Diasporafilm vermag es dabei, lokale, regionale und diasporische Filmkulturen zu verändern. Die dritte, diagonale, Betrachtungsweise setzt sich aus einer Kombination der zwei vorangegangenen zusammen. Diasporafilm ist in dem Sinne transnational, dass er fixe Ideen von nationalen Filmkulturen in Frage stellt. Diese verändern sich konstant durch das Changieren zwischen zwei oder mehr (Film-)Kulturen. Rückschlüsse von filmischen Austauschprozessen ermöglichen so eine Konzeption von Diaspora als Region, in der Diasporafilm als mediales Bindeglied fungiert.

Die Frage nach dem Größenverhältnis, der Skala, ist dabei ein wichtiger Faktor. Anna-Katharina Hornidge und Katja Mielke meinen:
A relational perspective on space underlining not only the constructed character of social and physical spaces and , areas' as manifestations of power relations, but in addition the interdependence of multiple spatialities, such as places, scales, networks, distances, and mobilities. (14)

Claudia Derichs bekräftigt ferner: „The reciprocal relationship between space (area) and regimes that ,scale“ particular elements of empirical reality, as well as between macro-conditions and micro-processes“ (35). Die Vorrangigkeit von Skala gegenüber Raum kann durch die vorliegende Arbeit als neues Analysewerkzeug auch für eine filmwissenschaftliche Auseinandersetzung produktiv gemacht werden. Die Filmwissenschaftlerin Kathleen Newman bemerkt in ihrem Sammelbandbeitrag „Notes on Transnational Film Theory“ dazu: 
How a film instantiates the geopolitical imaginary of a particular historical time and place, whom the film addresses and from what geopolitical perspective, and what a film accomplishes as a narrative are understood to be the simultaneous operation of multiple scales. (3-4)

Sie hebt dabei die Vielfältigkeit und Gleichzeitigkeit des Filmes als geopolitische Imagination und Perspektive hervor. Zugleich ist ein offener und beweglicher Begriff der Region in diesem Sinne somit auch für filmische Austauschprozesse von Bedeutung:

\begin{abstract}
Areas once considered peripheral (that is, less developed countries, so-called Third World) are now seen as integral to the historical development of cinema. The assumption that the export of European and US cinema to the rest of the world, from the silent period onward, inspired only derivative image cultures has been replaced by a dynamic model of cinematic exchange. (4)
\end{abstract}

Die Zirkulation von Film, präziser von Diasporafilm, als globales Phänomen ist demnach, so hat die vorliegende Arbeit gezeigt, als dynamisches und mehrdirektionales Phänomen zu begreifen.

Diasporafilm-Festivals ${ }^{65}$ spielen hierbei im Allgemeinen und im Falle der iranischen Diaspora im Besonderen für die Sichtbar- und Präsentmachung eine zentrale Rolle, denn sie bilden laut Hamid Naficy ,a third space of diaspora in which Iranian filmmakers could finally find not only a personal voice but also a collective one" (The Globalizing Era 412). Hierbei sind das von Hossein Mahini 1993 gegründete International Exile Film Festival in Göteborg, das von Bassir Nassibi 1995 ins Leben gerufene Sinema-ye Azad in Saarbrücken, das 2003 gegründete Iranian Film Festival in Paris sowie das 2007 ins Leben gerufene Noor Film Festival in Los Angeles, das 2010 gegründete UK Iranian Film Festival in 
London und zu guter Letzt das 2012 etablierte Persian International Film Festival in Sydney zu nennen. Die Filmfestivalforschung setzt sich mit Filmen, die in Iran produziert wurden und Eingang in den Kreislauf großer Festivals fanden, zunehmend auseinander. So konstatiert die Kommunikationswissenschaftlerin Azadeh Farahmand einen direkten Zusammenhang zwischen der Etablierung einer nationalen Filmkultur - getragen vom Autorenkino Kiarostamis, Makhmalbafs, Panahis und Farhadis - und der ökonomischen Logik von Filmfestivals in Bezug auf Programmierung und Marketingstrategie: „The festival exposure of Iranian films influences the very processes of production and affects the visual look and narrative tendencies of films - hence reinforcing the generic qualities of the national cinema“ („Disentangling“ 267). Diese Strategien entspringen der permanenten Suche nach neuen, innovativen Filmen und Filmschaffenden, bei gleichzeitiger Beibehaltung von Kategorien nationaler Filmkulturen: „Festivals typically pay special attention to films that have escaped local censorship - thereby enhancing the perceived festival image as the forum to display the authentic local reality otherwise filtered by government censorship“ (266). Mit Filmen, die außerhalb Irans produziert wurden und Eingang sowohl in große Filmfestivals als auch in kleinere Diasporafilm-Festivals finden, hat sich die Filmfestivalforschung bislang allerdings nur wenig befasst ${ }^{66}$. Der Diasporafilm stellt indes für die von Azadeh Farahmand aufgestellte These eine schillernde Kategorie dar, insofern als ethnische, kulturelle und nationale Zuschreibungen zunehmend verwischt, hinterfragt und unterwandert werden. Diese Spannungsverhältnisse, zwischen der Programmierung in Spezialsektionen von großen Festivals und der Programmierung in Hauptsektionen von DiasporafilmFestivals, sollten daher für eine wissenschaftliche Auseinandersetzung verstärkt in den Blick genommen werden. Die Möglichkeit zu weiterführender Forschung besteht dabei nicht darin, die ethnische Selbstdarstellung letzterer Festivals (von 


\section{Post-Diasporafilm oder Postdiaspora-Film? Ein Fazit}

Iraner_innen für Iraner_innen) zu beleuchten, sondern das Phänomen zu adressieren, dass Diasporafilm nun in der gesellschaftlichen Mitte angekommen ist, denn was für große Festivalkreisläufe gilt, ist auch für kleine valide. So postuliert beispielsweise das 2001 gegründete International Diaspora Film Festival in Toronto seinen Einsatzauftrag wie folgt: „IDFF provides Toronto audiences with an opportunity to experience the cultural mosaic of the present world through the medium of cinema" ${ }^{\text {“67. }}$. Damit wird eine Entwicklung deutlich, die den Diasporafilm von einem Zustand des Dazwischen, des „interstitial mode“ (Naficy, An Accented Cinema 46-56), zu einem aktiven Akteur in der Auseinandersetzung mit kultureller Differenz im Sinne einer gesamtgesellschaftlichen Öffnung macht.

Angesichts dieser globalen Zirkulation kultureller und medialer Elemente lässt sich mit Filmwissenschaftler Malte Hagener die Frage stellen: „Wo ist Film (heute)?“ In seinem gleichnamigen Text konstatiert er im Zeitalter digitaler Netzwerke eine Rekonfiguration des Filmes: Zeitgenössischer Film, ob Blockbuster-Produktion oder Autorenkino, arbeitet nicht nur mit digitaler Technologie, sondern übernimmt damit auch schrittweise YouTube-, Smartphone- und GameÄsthetiken, die so Teil des Filmes werden beziehungsweise im Umkehrschluss, den Filmwissenschaftlerinnen Shane Denson und Julia Leyda zufolge, diese Elemente bereits als filmisch ausweisen: „The aesthetic boundaries between arthouse film and blockbuster have become increasingly blurred as the mechanisms and perspectives of classical continuity are formally and materially challenged by a post-cinematic media regime“ (4). Die Veränderungen des Filmes als materielles Objekt photochemischer Aufnahmen und als textuelles (Kunst-)Werk sowie des Kinos als kulturelle und architektonische Institution im digitalen Zeitalter führten infolgedessen zu ,the emergence of a new, properly post-cinematic 
media regime“ (6). Post-Cinema meint in diesem Zusammenhang keine Kumulation neuer medialer Formen, stattdessen hilft der Begriff, so Shane Denson und Julia Leyda weiter, diese medialen Phänomene als Regionen und Landschaften zu begreifen: ,the term has a clear advantage in that it helps us to recognize this environment as a landscape, rather than merely a jumbled collection of new media formats, devices, and networks“ (1; Hervorhebung im Original). Dabei ginge es nicht um einen Paradigmenwechsel, sondern durchaus darum, filmische Transformationsprozesse im Lichte historischer Entwicklungen und Kontinuitäten zu sehen: „Post-cinema asks us to think about new media not only in terms of novelty but in terms of an ongoing, uneven, and indeterminate historical transition “ (2; Hervorhebung im Original). Ferner ermögliche der Begriff des PostCinema eine Sicht, die mediale (Um-)Formungen als ineinandergreifende kulturelle Phänomene auffasst: „Post-cinematic media are in conversation with and are engaged in actively re-shaping our inherited cultural forms, our established forms of subjectivity, and our embodied sensibilities“ (2). Post-Cinema bedeutet also im weitesten Sinne Bewegung, Übergang, Transformation, Verschränkung und Verflechtung:
A transitional movement taking place along an uncertain timeline, following an indeterminate trajectory, and charac- terized by juxtapositions and overlaps between the tech- niques, technologies, and aesthetic conventions of ,old ' and ,new' moving image media. (Denson und Leyda 6)

Film ist damit nicht mehr nur Kino, sondern Bewegtbild und Bildwelt in einer Fülle und Ballung, die kulturelle Verortung und Kommunikation ermöglicht:

Today, film is no longer just cinema, [...]. Film has become a purveyor of image worlds and virtual worlds of seemingly unlimited scope. It is no longer an art form celebrated only at public screenings, but a medium of cultural expres- 
sion easily attainable through informal communication. (Hagener, Hediger und Strohmaier 9)

Die Infragestellung von nationalen/kulturellen Grenzen und das damit verbundene Konzept der dynamischen Region eröffnet dabei die Möglichkeit für Aushandlungsprozesse, die nicht zuletzt auf einer wechselseitigen Kommunikationsleistung beruhen: „Different types of borders and boundaries are negotiated, take on shape, come into being, or are deconstructed again in and as a consequence of human interaction, which is also always communicative interaction" (Hornidge und Mielke 14). Diesen Argumentationslinien folgend ist Diaspora als dynamische kulturelle Region in der Welt, der Diasporafilm die kommunikative Interaktion ihrer Verflechtung. In diesem Zusammenhang kann Film als eine solche kommunikative Interaktion verstanden werden, die transregionale Verflechtung nicht nur forciert, sondern auch deren künstlerisch-ästhetischer Ausdruck ist. Mit den Worten Malte Hageners ausgedrückt:

Der Film ist mehr als je zuvor zu einer Lingua franca geworden, die überall auf der globalisierten Welt verstanden wird und damit Geltung und Relevanz beanspruchen kann. Film ist ein Medium der Kommunikation - wie Sport, Kochen oder Mode -, in dem Individuen und Gemeinschaften Kontakt miteinander und mit Außenstehenden eingehen. $(50-51)$

Will Higbee und Song H. Lim plädieren ihrerseits für:

A critical understanding of the political imbalances as well as the unstable and shifting identifications between host/home, individual/community, global/local and, indeed, national/transnational, as well as the tensions these generate within diasporic films. (12) 
In diesem Sinne ist der Diasporafilm, weg von Engführungen ethnischer $\mathrm{Zu}$ schreibungen, eine kulturelle Praxis, die filmische Räume und Filmwelten schafft. Ergo ist Diasporafilm nicht für das Verständnis einzelner ethnischer Gruppen relevant, sondern für ein erweitertes Verständnis aktueller Filmdynamiken per se unabdingbar.

Die von mir herausgearbeiteten neuen diasporafilmischen Räume zeugen von diesen Dynamiken. Khachig Tölölyan bemerkte: „There is indeed no place called diaspora, though there are sites of habitation and memory“ („Diaspora Studies“ 9). Michel Laguerre seinerseits spricht in seiner jüngsten Monographie The Postdiaspora Condition: Crossborder Social Protection, Transnational Schooling, and Extraterritorial Human Security von „Postdiaspora“ und meint damit ,the situation of individuals who live in a polity other than that into which they or their parents were born - their hostland - but possess full citizenship rights in their ancestral country - their homeland - similar or identical to those enjoyed by its intramural population“ (20). Er spricht damit eine Entwicklung an, die eine Fortführung und Weiterentwicklung von Diaspora meint. Er bezieht sich dabei auf die doppelte Staatsbürgerschaft von Menschen und die damit verbundenen sozioökonomischen und kulturellen Austauschprozesse. Der Begriff der Diaspora würde seiner Meinung nach gesellschaftliche Ausgrenzung und Marginalisierung bedeuten, jener der Postdiaspora hingegen ihre Überwindung:

Postdiaspora is an emancipatory move, refuting not the connection with one's place of origin, but rather one's unequal status vis-à-vis homelanders, and hostlanders. Furthermore, postdiaspora is the struggle to overcome an imposed status position based on place of origin. Postdiaspora 
does not mean marginalization in the way diaspora does, but rather difference. (22)

Diese Überwindung geschehe durch vier verschiedene Modelle: „Continuity model“, „,rupture and re-annexation model“, „,repatriation model“" und „postethnic model“, denen er ein fünftes Modell entgegensetzt, das „,cosmonational model“" (25-28). Wenn nun also die in dieser Arbeit analysierten diasporafilmischen Räume als Weiterentwicklung bereits bestehender filmischer Räume zu sehen sind, die zunehmend kulturelle und filmische Zuschreibungen verunmöglichen, dann kann möglicherweise von Postdiaspora-Film gesprochen werden, einer Spielart dessen, was Jeffrey Sconce in seinem Zeitschriftenartikel „Irony, Nihilism and the New American ,Smart' Film“ smart cinema nannte:

While previous forms of art cinema concentrated on formal experimentation with film style and narrative structure as a means of critiquing the codes of ,bourgeois realism "and/or ,bourgeois society', the new smart cinema has for the most part re-embraced classical narrative strategies, instead experimenting with tone $[\ldots]$ (352; Hervorhebung im Original).

Er zeichnet dabei eine historische Entwicklung nach, die Filme zunehmend als individuellen Ausdruck kultureller Positionierung und Verortung ansieht:

Smart cinema has displaced the more activist emphasis on the ,social politics' of power, institutions, representation and subjectivity so central to 1960s and 1970s art cinema (especially in its ,political' wing), and replaced it by concentrating, often with ironic disdain, on the ,personal politics' of power, communication, emotional dysfunction and identity in white middle-class culture. (352) 
Dabei fungiert sein Begriff eher als Sammelkategorie für eine Vielzahl an ästethischen und narrativen Formen, die Ausdruck einer je individuellen Handschrift sind: „Smart cinema should be seen as a shared set of stylistic, narrative and thematic elements deployed in differing configurations by individual films" (358). Der Diasporafilm ist also ein intelligentes Kino, welches kulturelle Differenz nicht nur repräsentiert und darstellt, sondern als Post-Diasporafilm möglicherweise bereits eine Überwindung dessen ist.

Als Forschungsgegenstand ficht der Diasporafilm zu guter Letzt auch die eigene wissenschaftliche Position als in westlichen Wissenschaftsstrukturen sozialisierte Film- und Medienwissenschaftlerin an: Wer spricht von welcher Position aus und in welcher Form über was oder wen? Die Beschäftigung mit Diasporafilm verlangt zunehmend nach neuen theoretischen Ansätzen und methodischen Zugängen. Die in der vorliegenden Arbeit postulierte Überwindung von Ethnozentrismus und Kulturessentialismus muss demnach auch mit einer kritischen Hinterfragung der eigenen eurozentristischen Forschungspraxis einhergehen.

Open Access Dieses Kapitel wird unter der Creative Commons Namensnennung 4.0 International Lizenz (http://creativecommons.org/licenses/by/4.0/deed.de) veröffentlicht, welche die Nutzung, Vervielfältigung, Bearbeitung, Verbreitung und Wiedergabe in jeglichem Medium und Format erlaubt, sofern Sie den/die ursprünglichen Autor(en) und die Quelle ordnungsgemäß nennen, einen Link zur Creative Commons Lizenz beifügen und angeben, ob Änderungen vorgenommen wurden.

Die in diesem Kapitel enthaltenen Bilder und sonstiges Drittmaterial unterliegen ebenfalls der genannten Creative Commons Lizenz, sofern sich aus der Abbildungslegende nichts anderes ergibt. Sofern das betreffende Material nicht unter der genannten Creative Commons Lizenz steht und die betreffende Handlung nicht nach gesetzlichen Vorschriften erlaubt ist, ist für die oben aufgeführten Weiterverwendungen des Materials die Einwilligung des jeweiligen Rechteinhabers einzuholen.

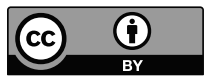

